\title{
Non-random distribution of high density chromatin detected at opposite ends of T-banded human metaphase chromosomes
}

\author{
Federico F. Santiñaque and Máximo E. Drets \\ Laboratory of Quantitative Microscopy, Instituto de Investigaciones Biológicas Clemente Estable, \\ Montevideo, Uruguay.
}

\begin{abstract}
Previous research using microdensitometric scanning and computer graphic image analysis showed that T-banded segments of human metaphase chromosomes usually exhibit an asymmetrical distribution of high density (HD) chromatin between sister chromatids. Here, we employed the same methods to analyze HD chromatin distribution at opposite ends of T-banded human lymphocyte chromosomes. This study revealed that in most chromosomes with an asymmetrical distribution of HD chromatin at both ends, the highest densities of each arm were located in opposite chromatids. The frequency of this configuration was 0.792 per chromosome, indicating that the highest chromatin densities of the terminal segments of T-banded human chromosomes were non-randomly distributed at opposite chromosome arms. The possible relationship of this observation to the mode of replication of the terminal chromosome region is briefly discussed.
\end{abstract}

Key words: human chromosomes, T-banding, chromatin structure, telomere, microdensitometry.

Received: November 6, 2006; Accepted: June 11, 2007.

It is well known that the usual banding procedures (C-, G-, R- and T-) reveal the underlying structure and composition of DNA and associated proteins in mitotic chromosomes (Therman and Susman, 1993). The specialized chromatin structure and the GC-richness of the telomeric/ subtelomeric DNA (de Lange, 2005; Riethman et al., 2004) confer high resistance to heat denaturation to the terminal regions of metaphase chromosomes which results in selective staining after T-banding (Dutrillaux, 1973). T-banding is a modification of R-banding procedure and is obtained through the incubation of chromosomes in a buffer at a high temperature followed by Giemsa staining. This procedure results in T-bands as darkly stained segments in lightly stained chromosomes. Scanning electron microscopy showed that T-bands are areas of highly aggregated chromatin fibres (Jack et al., 1986; Allen et al., 1988).

Microdensitometric scanning and computer graphic image analysis of high-resolution microphotographs of Tbanded segments of human and Chinese Hamster Ovary (CHO) chromosomes allowed the detection of a differential distribution of HD sub-telomeric chromatin between sister chromatids. The different patterns observed were arbitrarily classified in three kinds of HD chromatin distribution, namely: Type I, HD segments were of similar size in

Send correspondence to Federico F. Santiñaque. Instituto de Investigaciones Biológicas Clemente Estable, Av. Italia 3318, 11.600 Montevideo, Uruguay. E-mail: fsm@iibce.edu.uy. both sister chromatids; Type II, HD predominated in one of the chromatids; and Type III, HD was detected in only one chromatid (Drets et al., 1992). Alternatively, the distribution of HD chromatin could be classified in a simplified way as symmetrical (pattern type I) or asymmetrical (patterns type II and III). These patterns were confirmed by scanning T-banded endoreduplicated $\mathrm{CHO}$ chromosomes in which the same interchromatid distribution of HD chromatin appeared in both sister chromosomes (Drets and Mendizábal, 1998). Nevertheless, quantitative computerized microdensitometrical analyses on the distribution of HD chromatin at opposite ends of T-banded chromosomes have not yet been reported. Therefore, we performed microdensitometric scanning and computer graphic image analysis to study HD chromatin distribution at T-banded human chromosomes that showed asymmetry at opposite ends.

Human chromosomes were obtained from peripheral lymphocytes using routine methods. Dutrillaux's T-banding method was used with minor modifications (Drets et al., 1992). T-banded chromosomes were photographed under a Zeiss Photomicroscope II using a Neofluar oil immersion objective (100X), double immersion and phase contrast. Technical Pan film (Kodak) was exposed at 12 DIN and developed with Microdol-X (Kodak) at $20{ }^{\circ} \mathrm{C}$ for nine minutes. Negatives were enlarged using a DA900 Durst Professional enlarger with a Componon lens (NA 
1:3.5, Schneider) on Fine Grain Positive Film (Kodak) and developed with Dektol (Kodak) for one minute at $20^{\circ} \mathrm{C}$. Twenty five metaphases with a large number of T-banded chromosomes were photographed. Well spread chromosomes with evident T-bands at both ends were selected for microdensitometric scanning. Chromosomes pairs 13, 14, $15,19,20,21,22$ and the Y chromosome were excluded from the analyses because of their small short arm and length which prevent a detailed quantitative analysis of the terminal chromosome region. Our study involved the microdensitometric scanning of approximately 1000 chromatid ends corresponding to $250 \mathrm{~T}$-banded chromosomes. A microscope photometer MSP65 system (Zeiss) equipped with a fast scanning stage $(0.25 \mu \mathrm{m} / \mathrm{step})$, a Zeiss Luminar

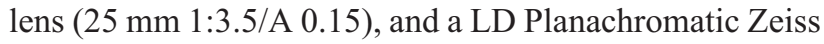
condenser objective (40/0.60-160/1.1-1.5) was used for scanning T-banded human chromosomes photomicrographs. The MSP65 microprocessor was connected with a DEC Micro-VAX 3300 computer (Digital) and a TEK Netstation 4211 (Tektronix) computer graphic color terminal which was used for generating quantitative images from the microdensitometric data. All chromosome scannings covered an object area of $100 \times 100$ stage steps, totaling 100 absorbance measurements per step that were averaged in order to reduce any eventual random noise produced by the electronic components. Averaged measurements were displayed into two dimensional image units (pixels) and sorted into twelve density intervals ranging from 0 to 100 absorbance units. Bicubic interpolation was used to increase image resolution allowing a more detailed visualization of the chromosome structure. Chromosome graphic images were photographed directly from the TEK Netstation terminal. The computer procedures used were reported elsewhere (Drets et al., 1995).

Microdensitometric scanning and computer graphic image analysis produced quantitative images that closely corresponded to the chromosome morphology observed under the light microscope. However, the high magnification of the terminal chromosome region obtained with this method enabled the detection of subtle staining density differences, such as symmetric and asymmetric patterns of HD chromatin distribution, undetectable with light microscopy. We studied the distribution of the highest chromatin densities in chromosomes that exhibited asymmetric patterns in both ends. Thus, each T-banded human chromosome was first entirely scanned, which was followed by an individual scanning of both chromosome ends in order to get a more detailed quantitative image of the interchromatid distribution of HD chromatin. Counting of the number of pixels in each HD area through a graphic computational procedure provided an arbitrary quantitative criterion to discriminate between symmetrical and asymmetrical patterns. We considered as symmetrical those interchromatid differences lower than 25 percent and as asymmetrical differences higher than 25 percent between sister chromatids. Chromosomes that exhibited T-banded segments with a type I density pattern or HD areas presenting minute sister chromatid exchanges were not considered in our analysis.

Two configurations were detected in chromosomes presenting asymmetry at both ends: the highest densities of each chromosome arm were either located in opposite chromatids (TRANS; Figure 1a) or in the same chromatid (CIS; Figure 1b). Microdensitometric analyses of opposite ends of T-banded human chromosomes revealed that of a total of 101 scanned chromosomes with an asymmetrical distribution of HD chromatin between sister chromatids in both arms, 80 had a TRANS configuration and 21 had a CIS configuration (Figure 1). The frequencies of each configuration per chromosome (TRANS: 0.792; CIS: 0.208) were considerably different than expected (Chi-square test, $\mathrm{p}<<0.001$ ), indicating that the highest chromatin densities of the terminal segments of T-banded human chromosomes appeared non-randomly located at opposite chromosome arms. It is of interest to mention that we have recently reported similar observations in T-banded CHO chromosomes (Drets and Santiñaque, 2007).

T-banded segments of human and CHO chromosomes exhibit a complex distribution of HD chromatin between sister chromatids (Drets et al., 1992). The detection of similar patterns in endoreduplicated T-banded $\mathrm{CHO}$ chromosomes through microdensitometric scanning and computer graphic image analysis indicated that the differential distribution of HD chromatin was not due to a methodological artifact since it replicated in the same manner in the terminal region of both sister chromosomes (Drets, 2000). Moreover, T-banded segments of human and CHO chromosomes sometimes exhibited HD chromatin areas distributed like minute sister chromatid exchanges (Drets et al., 1992), what we recently called ter-SCE (Drets et al., 2006). In this work we showed that HD chromatin was non-randomly distributed at opposite ends of T-banded human chromosomes, with the highest chromatin densities located in a TRANS configuration in most chromosomes. These observations suggest that a normal phenomenon occurring in the terminal chromosome region may be involved.

Significant differences in telomeric signal intensities between sister chromatids of human metaphase chromosomes were reported using fluorescent labelled telomeric peptidic nucleic acid (PNA) probes and three-dimensional confocal microscopy (Bekaert et al., 2002). These findings conform with the differential distribution of HD chromatin observed in T-banded human chromosomes. This may indicate that the highly heat-resistant HD areas could interfere with DNA accessibility to telomere probes. In human metaphase chromosomes, HD chromatin T-banded areas co-localize with fluorescent signals of FITC-labeled telomere PNA probes (CCCTAA), which are non-strictly localized at the end of the chromatids (Drets, 2004; Moyzis et al., 

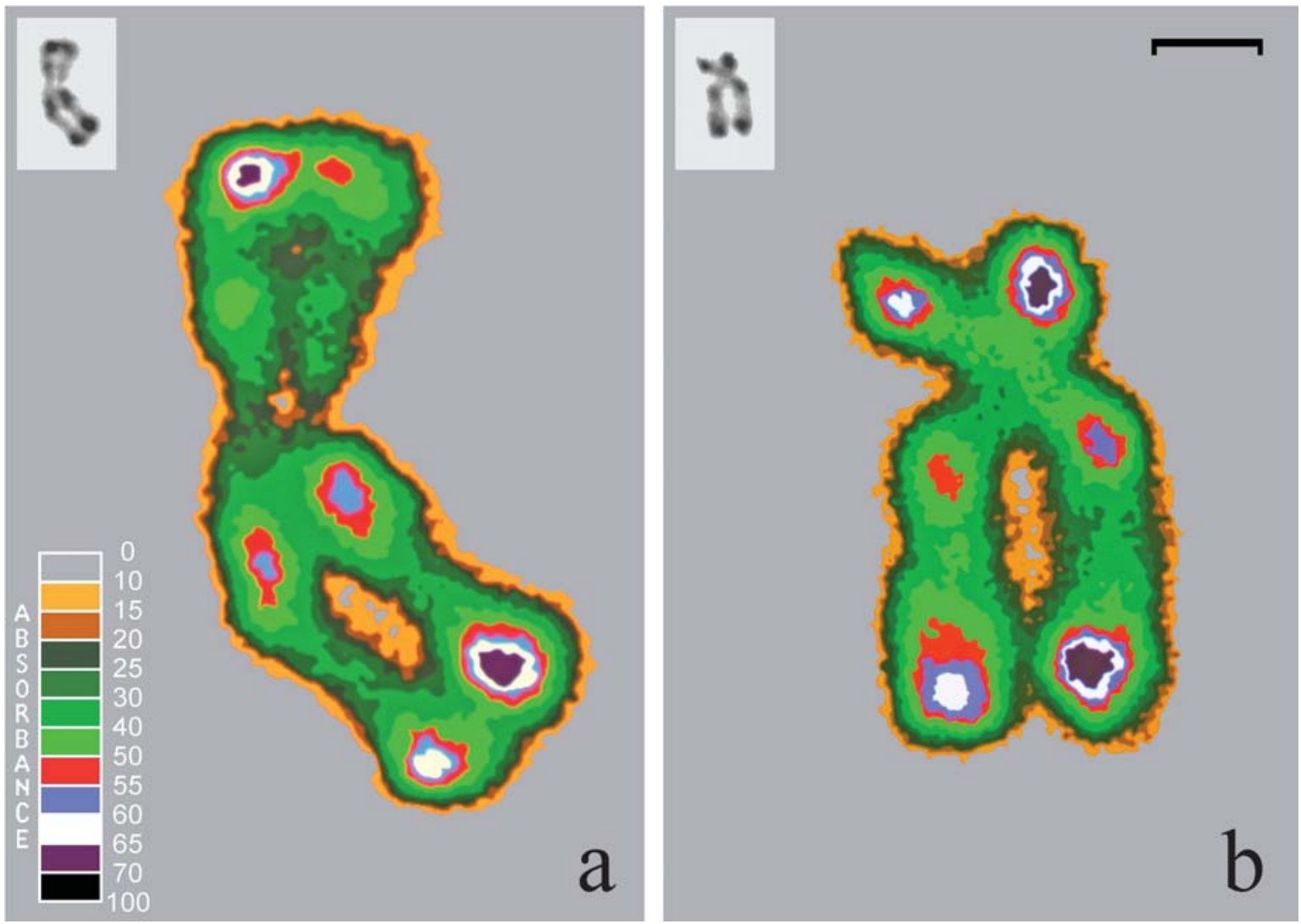

Figure 1 - Graphic images of T-banded human chromosomes showing TRANS (a) and CIS (b) configurations obtained through microdensitometric scanning and computer graphic image analysis. Remnants of R-bands are still observed since T-bands are a sub-set of them. Insets illustrate microscopic images of the scanned T-banded chromosomes. Bar $=1 \mu \mathrm{m}$.

1988; Steinmüller et al., 1993). Interestingly, the TRANS and CIS configurations frequencies found here are comparable to those obtained in normal human mitotic chromosomes using telomeric CO-FISH, a method that allows to differentiate between telomeres that replicated through lagging- or leading-strand synthesis (Cornforth and Eberle, 2001). These authors suggested that the CIS configuration could be explained in several ways: (a) by the ocurrence of an odd number of sister chromatid exchanges along the chromosome, (b) by an overlap of sister chromatids near the centromeric region, and, (c) by spontaneous recombination in the subtelomere, that resulted in TRANS to CIS pattern conversions.

Although the molecular analysis of the terminal chromosome region is producing considerable data about its structure and function, we have no explanation for our observations. Since the phenomenon that we detected in normal T-banded chromosomes has a similar frequency to that obtained using telomeric CO-FISH and that HD chromatin areas colocalize with telomeric DNA, we suppose that they may be related to the specialized high-order chromatin structure nucleated by the telomeric TRF2, TRF1, POT1, TIN2, TPP1 and Rap1 proteins (de Lange, 2005). Accordingly, our observations could reflect a non-random segregation of some telomeric components of the HD chromatin to only one sister telomere during its replication. The chromatin in this telomere would be more compact than in the other telomere which would later incorporate those components to nucleate the HD chromatin state. This agrees with recent findings showing that sister telomeres are unequally susceptibile to the transient expression of dominant mutants of the protective protein TRF2, that leads to deletions or end-to-end chromatid-type fusions in a TRANS configuration involving only leading-strand telomeres (Bailey et al., 2001; Bailey and Murnane, 2006; Wang et al., 2004). Additional experimental data are extremely important to test this hypothesis since other possibilities cannot be excluded at present. Thus, a combined molecular and cytogenetic analysis would provide a better understanding of the high-order chromatin organization and replication of the terminal chromosome region.

\section{Acknowledgments}

Supported in part by the Program for Development of Basic Sciences (PEDECIBA), Uruguay.

\section{References}

Allen TD, Jack EM and Harrison CJ (1988) The three-dimensional structure of human metaphase chromosomes deter- 
mined by scanning electron microscopy. In: Adolph KW (ed) Chromosomes and Chromatin. CRC Press, Boca Raton, pp 51-72.

Bailey SM, Cornforth MN, Kurimasa A, Chen DJ and Goodwin EH (2001) Strand-specific postreplicative processing of mammalian telomeres. Science 293:2462-2465.

Bailey SM and Murnane JP (2006) Telomeres, chromosome instability and cancer. Nucleic Acids Res 34:2408-2417.

Bekaert S, Koll S, Thas O and Van Oostveldt P (2002) Comparing telomere length of sister chromatids in human lymphocytes using three-dimensional confocal microscopy. Cytometry 48:34-44.

Cornforth MN and Eberle RL (2001) Termini of human chromosomes display elevated rates of mitotic recombination. $\mathrm{Mu}-$ tagenesis 16:85-89.

de Lange T (2005) Shelterin: The protein complex that shapes and safeguards human telomeres. Genes Dev 19:2100-2110.

Drets ME (2000) Insights into the structure of the subtelomeric chromosome segments. Genet Mol Biol 23:1087-1093.

Drets ME (2004) Cytological indications of the complex subtelomeric structure. Cytogenet Genome Res 104:137-141.

Drets ME and Mendizábal M (1998) The underlying structure of the subtelomeric segments detected by microphotometrical scanning and graphic image analysis. Mut Res 404:13-16.

Drets ME and Santiñaque FF (2007) A biomedical perspective of the telomeric structure and function. In: Obe $\mathrm{G}$ and Vijayalaxmi (eds) Chromosomal Alterations: Methods, Results and Importance in Human Health. Springer-Verlag, Berlin Heidelberg, pp 223-238.

Drets ME, Obe G, Monteverde FJ, Folle GA, Medina II, De Galvez MG, Duarte JE and Mechoso BH (1992) Computerized graphic and light microscope analyses of T-banded chromosome segments of Chinese hamster ovary cells and human lymphocytes. Biol Zentrbl 111:204-214

Drets ME, Drets GA, Queirolo PJ and Monteverde FJ (1995) Computer graphics as a tool in cytogenetic research and education. Comp Appl Biosc (CABIOS) 11:463-468.

Drets ME, Santiñaque FF and Obe G (2006) A proposal of a standardised nomenclature for terminal minute sister chromatid exchanges. Genet Mol Biol 29:446-447.

Dutrillaux B (1973) Nouveau système de marquage chromosomique: Les bandes T. Chromosoma 41:395-402.

Jack EM, Harrison CJ and Allen TD, Harris R (1986) A structural basis for R- and T-banding: A scanning electron microscopy study. Chromosoma 94:395-402.

Moyzis RK, Buckinham JM, Cram LS, Dani M, Deaven LL, Jones MD, Meyne J, Ratliff RL and Wu JR (1988) A highly conserved repetitive DNA sequence (TTAGGG)n present at the telomeres of human chromosomes. Proc Natl Acad Sci USA 85:6622-6626.

Riethman H, Ambrosini A, Castaneda C, Finklestein J, Hu XL, Mudunuri U, Paul S and Wei J (2004) Mapping and initial analysis of human subtelomeric sequence assemblies. Genome Res 14:18-28.

Steinmüller J, Schleiermacher E and Scherthan H (1993) Direct detection of repetitive whole chromosome paint and telomere DNA probes by immunogold electron microscopy. Chromosome Res 1:45-51.

Therman E and Susman M (1993) Human Chromosomes: Structure, Behavior and Effects. 3rd edition. Springer-Verlag, New York, 376 pp.

Wang RC, Smogorzewska A and de Lange T (2004) Homologous recombination generates T-loop-sized deletions at human telomeres. Cell 119:355-368.

Associate Editor: Peter L. Pearson 